\title{
Cytochrome P450 2A5
}

National Cancer Institute

\section{Source}

National Cancer Institute. Cytochrome P450 2A5. NCI Thesaurus. Code C18024.

Mouse cytochrome P450 2a5 protein (494 aa, 57 kD) is encoded by the mouse Cyp2a5 gene. This enzyme is an unspecific, membrane-bound, monooxygenase located in the endoplasmic reticulum. It catalyzes the metabolism of a variety of substrates including fatty acids, steroids and xenobiotics. 